\title{
Efecto anti-Trypanosoma cruzi del aceite esencial de Cymbopogon citratus (DC) Stapf (hierba luisa) en ratones Balb/c Cymbopogon citratus (DC) Stapf (lemongrass) essential oil has anti-Trypanosoma cruzi effect in Balb/c mice
}

\author{
Juan Rojas ${ }^{1}$, Sergio Ronceros ${ }^{1}$, Olga Palacios ${ }^{2}$, Carlos Sevilla ${ }^{2}$ \\ Instituto de Investigaciones Clinicas, Facultad de Medicina, Universidad Nacional Mayor de San Marcos, Lima, Perú. \\ ${ }^{2}$ Instituto de Medicina Tropical, Facultad de Medicina, Universidad Nacional Mayor de San Marcos, Lima, Perú.
}

\begin{abstract}
Resumen
Objetivo: Determinar la actividad anti-Trypanosoma cruzi in vivo del aceite esencial de Cymbopogon citratus en ratones Balb/c. Diseño: Estudio experimental, prospectivo. Institución: Instituto de Investigaciones Clínicas e Instituto de Medicina Tropical de la Facultad de Medicina de la Universidad Nacional Mayor de San Marcos, Lima, Perú. Material biológico: Aceite esencial de Cymbopogon citratus; ratones albinos Balb/c. Intervenciones: Los animales fueron asignados aleatoriamente a seis grupos de 15 ratones cada uno: infectados y no tratados (G1), infectados y tratados con benznidazol $100 \mathrm{mg} / \mathrm{kg}\left(\mathrm{G}_{2}\right)$, infectados y tratados con aceite esencial de Cymbopogon citratus $100 \mathrm{mg} / \mathrm{kg}$ y $250 \mathrm{mg} / \mathrm{kg}$ (G3 y G4, respectivamente), no infectados y no tratados (G5), y no infectados y tratados con $250 \mathrm{mg}$ de Cymbopogon citratus (G6). La infección con T. cruzi se realizó con $10^{4}$ trypomastigotes sanguíneos y el tratamiento empezó en el $8^{\circ}$ dia post infección (dpi) hasta el $28^{\circ} \mathrm{dpi}$. La parasitemia se determinó con microscopia óptica cada dos días en $5 \mu \mathrm{L}$ de sangre de la cola. En el $14^{\circ}, 21^{\circ}$ y $28^{\circ} \mathrm{dpi}$, cinco animales de cada grupo fueron sacrificados y se removió el corazón para estudio histopatológico. Principales medidas de resultados: Parasitemia, número de nidos de amastigotes e infiltrados inflamatorios. Resultados: El aceite esencial de Cymbopogon citratus $250 \mathrm{mg} / \mathrm{kg} /$ dia produjo una reducción significativa en el pico de parasitemia desde 113,92 $\pm 25,66$ hasta $74,60 \pm 12,37$ tripomastigotes $/ \mathrm{mL}(p<0,05)$. Con $100 \mathrm{mg} / \mathrm{kg} /$ dia se produjo una reducción hasta $77,40 \pm 14,93$ tripomastigotes/ $\mathrm{mL}(\mathrm{p}<0,05)$. También redujo el número de amastigotes y de infiltrados inflamatorios en el corazón. Conclusiones: El aceite esencial de Cymbopogon citratus tuvo efecto anti-Trypanosoma cruzi en ratones Balb/c en lo referente a la disminución de la parasitemia, el número de nidos de amastigotes y los resultados inflamatorios.
\end{abstract}

Palabras clave: Trypanosoma cruzi, Cymbopogon citratus, hierba luisa, aceite esencial.

Abstract

Objective: To determine anti-Trypanosoma cruzi effect of Cymbopogon citratus essential oil in Balb/c mice. Design: Experimental, prospective study. Setting: Institute of Clinical Research and Institute of Tropical Medicine, Faculty of Medicine, Universidad Nacional Mayor de San Marcos, Lima, Peru. Biological material: Cymbopogon citrates essential oil; Balb/c albino mice. Interventions: Animals were randomly assigned to six groups ( $n=15)$ : infected and untreated $(\mathrm{G} 1)$, infected and treated with benznidazole $100 \mathrm{mg} / \mathrm{kg}(\mathrm{G} 2)$, infected and treated with essential oil of Cymbopogon citratus $100 \mathrm{mg} / \mathrm{kg}$ and $250 \mathrm{mg} / \mathrm{kg}$ (G3 and G4, respectively), uninfected and untreated (G5), and uninfected and treated with $250 \mathrm{mg} / \mathrm{kg}$ of Cymbopogon citratus (G6). Infection with T. cruzi was conducted with $10^{4}$ blood trypomastigotes and treatment began on 8th day post infection (dpi) through 28 th dpi. Parasitemia was determined by optical microscopy every 2 days in $5 \mu \mathrm{L}$ of blood drawn from the tail. In dpi 14, 21 and 28, five animals from each group were sacrificed and the heart was removed for pathology study. Main outcome measures: Parasitemia, number of amastigotes nests and inflammatory infiltrates. Results: Cymbopogon citratus essential oil $250 \mathrm{mg} / \mathrm{kg} /$ day reduced parasitemia peak significantly from $113.92 \pm 25.66$ to $74.60 \pm 12.37$ tripomastigotes $/ \mathrm{mL}(p<0.05)$. With $100 \mathrm{mg} / \mathrm{kg} /$ day there was a reduction to $77.40 \pm 14.93$ tripomastigotes $/ \mathrm{mL}$ ( $p$ $<0.05$ ). There was also reduction in number of amastigotes and inflammatory infiltrates in the heart. Conclusions: Cymbopogon citratus essential oil had anti-Trypanosoma cruzi effect in Balb/c mice as shown by decline in parasitemia, number of amastigotes nests and inflammatory infiltrates.

Key words: Trypanosoma cruzi, Cymbopogon citratus, lemongrass, essential oil.

An Fac med. 2012;73(1):7-12 


\section{INTRODUCCIÓN}

La tripanosomiasis americana o enfermedad de Chagas está presente en casi todos los países de América Latina y afecta de 18 a 20 millones de personas, desde México hasta Chile y Argentina. En el Perú, los departamentos en los que se ha reportado casos autóctonos de enfermedad de Chagas son Piura, Cajamarca, Amazonas, Apurímac, San Martín, Junín, Ucayali, Huánuco, Ica, Arequipa, Moquegua y Tacna ${ }^{(1)}$.

La farmacoterapia actual de la enfermedad de Chagas comprende escasamente dos fármacos, el nifurtimox y el benznidazol; ambos son tóxicos, es necesario administrarlos por largo tiempo y solo sirven para la fase aguda de la enfermedad (2). Ante esta situación, es urgente la búsqueda de nuevos agentes terapéuticos para la enfermedad de Chagas y las plantas medicinales son una alternativa viable.

Cymbopogon citratus (DC) Stapf es una hierba de la familia Poaceae (Gramineae), nativa del Sureste de Asia, pero actualmente crece alrededor del mundo, principalmente en las regiones tropicales y sabanas. Conocida con el nombre común, según los diversos países, de 'hierba luisa', 'caña santa', 'lemongrass', 'te limón', 'citronela' y otros, es una planta perenne que crece formando densos grupos de hasta $3 \mathrm{~m}$ de altura, con rizomas cortos. Sus hojas son erectas, glabras planas, de más de 1 $\mathrm{m}$ de largo, $5 \mathrm{a} 15 \mathrm{~mm}$ de ancho, borde superior cerrado en la base, con márgenes rugosos y lígulas membranosas o áridas de 4 a $5 \mathrm{~mm}$ de largo, inflorescencias erectas, generalmente en pares de racimos ${ }^{(3)}$.

En medicina tradicional, Cymbopogon citratus es utilizada en la forma de decocción o infusión como anticatarral, antiespasmódico, hipotensor, antipirético y tranquilizante ${ }^{(4,5)}$.

Los estudios farmacológicos realizados en diferentes modelos experimentales han demostrado varias propiedades de Cymbopogon citratus. Así, los diversos extractos preparados de esta planta han mostrado actividades como antioxidante ${ }^{(6-10)}$, hipocolesterolémica ${ }^{(11,12)}$, sedante ${ }^{(13)}$, antiinflamatoria ${ }^{(10,14,15)}$, hipoglicemiante ${ }^{(12)}$, antifúngica contra Epidermophyton flocosum, Trichophyton rubrum y Candida albicans ${ }^{(16,17)}$, inhibición de Staphylococcus aureus y Escherichia coli ${ }^{(18)}$ y sobre Salmonella typhi ${ }^{(19)}$, inhibición de la formación de focos de criptas aberrantes en el estadio de iniciación y promoción en el colon de ratas ${ }^{(20)}$, así como inhibición de la fase temprana de hepatocarcinogénesis en ratas ${ }^{(21)}$. Además, tres C-glicosilflavonoides de las hojas de Cymbopogon citratus mostraron efecto inhibitorio significativo de la oxidación de LDL, pudiendo ser beneficiosos para prevenir o atenuar la aterosclerosis ${ }^{(22)}$.

Por otra parte, el aceite esencial de Cymbopogon citratus también ha demostrado variadas actividades biológicas como antifúngica ${ }^{(23-25)}$, bactericida contra Helicobacter pylori ${ }^{(26)}$, antibacteriana contra Listeria innocua ${ }^{(27)}$, ansiolítico, sedativo y anticonvulsivante ${ }^{(28)}$, y anticonvulsivante, cuyo mecanismo de acción, al menos en parte, depende de la neurotransmisión GABAérgica ${ }^{(29)}$; analgésico ${ }^{(30)}$, larvicida, ovicida y repelente contra el mosquito de la filaria, Culex quinquefasciatus (31). Asimismo, el principal constituyente del aceite esencial, el citral, mostró significativo efecto antiinflamatorio, debido a la inhibición de la producción de óxido nítrico a través de la supresión de la actividad del NFkB ${ }^{(32)}$. También mostró potencial anticáncer al inducir apoptosis en varias líneas celulares de cáncer hematopoyético, acompañado de fragmentación del ADN e inducción de la actividad catalítica de la caspasa-3 ${ }^{\text {(33) }}$. Además, claramente inhibió el crecimiento tanto de micelios como de levaduras de Candida albicans (23).

La mayoría de estudios de la actividad tripanocida con derivados de plantas se ha efectuado solamente in vitro. En un estudio realizado por nuestro equipo de investigación, hemos demostrado que entre diez aceites esenciales de plantas medicinales, Cymbopogon citratus (DC) Stapt inhibió el crecimiento in vitro de epimastigotes de $\mathrm{T}$. cruzi, con una buena $\mathrm{CI}_{50}$ de 63,09 $\mu \mathrm{g} /$ $\mathrm{mL}{ }^{(34)}$. Esto nos motivó a continuar el estudio, por lo que nos propusimos evaluar in vivo el efecto anti-Trypanosoma cruzi del aceite esencial de Cymbopogon citratus en un modelo de ratón.

\section{MÉTODOS}

En el presente estudio experimental, prospectivo, se utilizó una cepa de Trypanosoma cruzi aislada del vector Triatoma infestans colectado en el Departamento de Arequipa, Perú. Los parásitos fueron mantenidos por pasajes de sangre infectada a ratones albinos Balb/c normales.

En el experimento se usó tripomastigotes sanguíneos cosechados por punción cardíaca de los ratones infectados por Trypanosma cruzi, en el pico de parasitemia.

Para la infección in vivo, se obtuvo ratones albinos machos $(25 \pm 2 \mathrm{~g})$ del Instituto Nacional de Salud, mantenidos bajo un ciclo de luz/oscuridad de 12/12 horas y se les permitió una semana de aclimatación antes del experimento, con alimento y agua ad líbitum. La infección se realizó por inyección intraperitoneal de $10^{4}$ tripomastigotes sanguíneos.

Los animales fueron asignados aleatoriamente a los siguientes grupos experimentales ( $n=15)$ : a) infectados y no tratados (G1); b) infectados y tratados con benznidazol $100 \mathrm{mg} / \mathrm{kg}$ (G2); c) infectados y tratados con aceite esencial de Cymbopogon citratus 100 $\mathrm{mg} / \mathrm{kg}(\mathrm{G} 3)$; d) infectados y tratados con aceite esencial de Cymbopogon citratus $250 \mathrm{mg} / \mathrm{kg}(\mathrm{G} 4)$; e) no infectados y no tratados $(G 5)$; y, f) no infectados y tratados con $250 \mathrm{mg}$ de Cymbopogon citratus (G6).

La planta Cymbopogon citratus (DC) Stapt fue colectada en el Jardín botánico de la Facultad de Medicina de la Universidad Nacional Mayor de San Marcos, Lima, Perú; una muestra fue 
llevada al Museo de Historia Natural de la Universidad Nacional Mayor de San Marcos para su identificación taxonómica (voucher: 069-USM-2010).

El aceite esencial de Cymbopogon citratus fue obtenido por arrastre con vapor de agua ${ }^{(35)}$, para lo cual se utilizó las hojas frescas de la planta; el destilado se separó tomando en cuenta sus propiedades de inmiscibilidad y diferencia de densidades entre el agua y el aceite esencial. Utilizando una pera de separación de vidrio, se deshidrató las impurezas de agua en el aceite esencial con $\mathrm{Na}_{2} \mathrm{SO}_{4}$ anhidro, se filtró, guardándose en un frasco de vidrio color ámbar bajo refrigeración, a una temperatura de $4 \stackrel{\circ}{ } \mathrm{C}$, hasta su uso. El aceite esencial fue disuelto en dimetilsulfóxido (DMSO) al 1\% y se administró por vía oral en las dosis indicadas a los grupos G3, G4 y G6, desde el $8^{\circ}$ al $28^{\circ}$ dpi (día post infección). El benznidazol fue administrado en dosis de $100 \mathrm{mg} / \mathrm{kg} / \mathrm{día}$ por vía oral (G2), en un esquema similar a los grupos anteriores, mientras que el G1 solo recibió el vehículo.

La parasitemia fue determinada individualmente cada dos días por microscopia directa, contando los parásitos en $5 \mu \mathrm{L}$ de sangre obtenida de la cola del ratón.

En los dpi 7, 14, 21 y 28, se evaluó el peso corporal.

En el $14^{\circ}, 21^{\circ}$ y $28^{\circ} \mathrm{dpi}$, cinco ratones de cada grupo fueron sacrificados por dislocación cervical y los corazones fueron rápidamente removidos y procesados para análisis histopatológico. Los corazones fueron cortados longitudinalmente, lavados en buffer fosfato salino (PBS) helado y fijados en formaldehido al 10\% en PBS. Los tejidos fijados fueron deshidratados y embebidos en parafina. Secciones de $3 \mu \mathrm{m}$ fueron coloreadas con hematoxilina-eosina (HE) y analizados por microscopia de luz. Se determinó el número de nidos de amastigotes y de infiltrados inflamatorios (más de 10 células mononucleares) en 100 campos en cada lámina. Se obtuvo el número promedio de nidos de amastigotes o infiltrados inflamato-

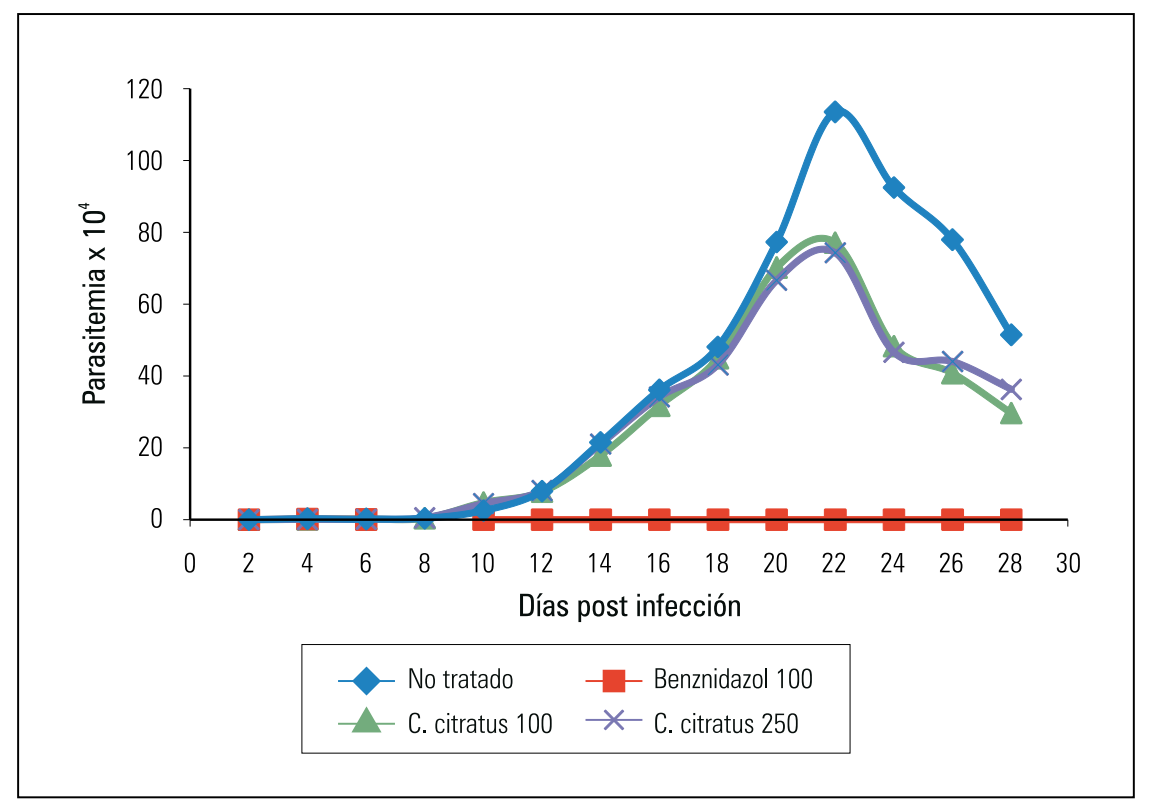

Figura 1. Efecto del tratamiento por vía oral con aceite esencial de Cymbopogon citratus sobre la parasitemia en ratones infectados con $10^{4}$ tripomastigotes sanguíneos de T. cruzi, durante 28 dias. En el pico de la parasitemia, en el 22 dpi, se produjo una reducción significativa con el tratamiento con C. citratus en dosis de 250 y $100 \mathrm{mg} / \mathrm{kg}(p<0,05)$, según análisis de datos por ANOVA de una vía seguido por una prueba post hoc de Scheffé.

rios por campo, con tres secciones de cada ratón.

Al inicio del experimento y en los dpi $7,14,21$ y 28 , se evaluó el peso corporal.

Los datos obtenidos fueron expresados como media \pm desviación estándar y las comparaciones entre los grupos experimentales fueron realizadas por ANOVA de una vía seguido por una prueba post-hoc de Scheffé. Se consideró las medias significativamente diferentes cuando $\mathrm{p}<0,05$. Se utilizó el software SPSS 15.0.

\section{RESULTADOS}

El tratamiento a partir del $8^{\circ} \mathrm{dpi}$ con el aceite esencial de Cymbopogon citratus (hierba luisa) en dosis de $250 \mathrm{mg} / \mathrm{kg} / \mathrm{d}$ áa por vía oral produjo una reducción significativa desde 113,92 $\pm 25,66$ hasta $74,60 \pm 12,37$ tripomastigotes $/ \mathrm{mL}$ (p $<0,05)$ en el pico de la parasitemia, en el $22^{\circ}$ dpi. Con la dosis de $100 \mathrm{mg} /$ $\mathrm{kg} /$ día se produjo una reducción de la parasitemia hasta $77,40 \pm 14,93$ tripomastigotes $/ \mathrm{mL}(\mathrm{p}<0,05)$. La parasitemia se redujo $100 \%$ con el tratamiento con $100 \mathrm{mg} / \mathrm{kg} / \mathrm{día}$ de benznidazol, tal como se muestra en la figura 1 .

La ganancia de peso corporal fue alterada levemente en los ratones infectados, pero la disminución del peso no fue significativa en ninguno de los grupos respecto al control (G5: no infectado-no tratado), ni siquiera en el dpi 28 (tabla 1).

El mayor número de nidos de amastigotes se observó en el $28^{\circ}$ dpi en el grupo infectado y no tratado $(7,60 \pm$ 2,48 nidos de amastigotes/100 campos), seguido por el grupo infectado y tratado con $100 \mathrm{mg} / \mathrm{kg}$ de Cymbopogon citratus $(2,00 \pm 1,55$ nidos de amastigotes/100 campos), produciendo dicho tratamiento una reducción de $73,68 \%$, mientras que con $250 \mathrm{mg} / \mathrm{kg}$ de Cymbopogon citratus se produjo una reducción significativa de $89,47 \%(p<0,05)$. En el grupo tratado con benznidazol $100 \mathrm{mg} /$ kg, no se observó nidos de amastigotes en el $28^{\circ}$ dpi (figura 2). 
Tabla 1. Variación del peso corporal de ratones infectados con 104 trypomastigotes de T. cruzi tratados con benznidazol y Cymbopogon citratus, comparado con ratones no infectados.

\begin{tabular}{cccccc} 
Grupo & \multicolumn{5}{c}{ Días post infección (dpi) } \\
\cline { 2 - 6 } Infectado-No tratado & 0 & 7 & 14 & 21 & 28 \\
Infectado-Benznidazol 100 & $28,33 \pm 3,44$ & $30,60 \pm 2,97$ & $31,47 \pm 3,85$ & $31,00 \pm 3,24$ & $31,40 \pm 3,65$ \\
Infectado-C. citratus 100 & $27,80 \pm 2,18$ & $31,40 \pm 2,26$ & $31,60 \pm 3,27$ & $32,20 \pm 2,68$ & $32,20 \pm 3,03$ \\
Infectado- C. citratus 250 & $28,67 \pm 2,50$ & $30,33 \pm 2,29$ & $31,13 \pm 2,13$ & $29,20 \pm 4,55$ & $29,80 \pm 5,21$ \\
No infectado-No tratado & $27,80 \pm 3,14$ & $30,67 \pm 3,77$ & $30,47 \pm 4,12$ & $30,40 \pm 4,93$ & $30,20 \pm 4,10$ \\
No infectado-C. citratus 250 & $28,73 \pm 2,09$ & $30,27 \pm 2,28$ & $31,00 \pm 2,59$ & $34,60 \pm 2,30$ & $36,20 \pm 2,77$ \\
\hline
\end{tabular}

Valores expresados como media aritmética \pm desviación estándar.

No existen diferencias significativas con respecto al grupo No infectado-No tratado. Datos analizados por ANOVA de una vía seguido por una prueba post hoc de Scheffé.

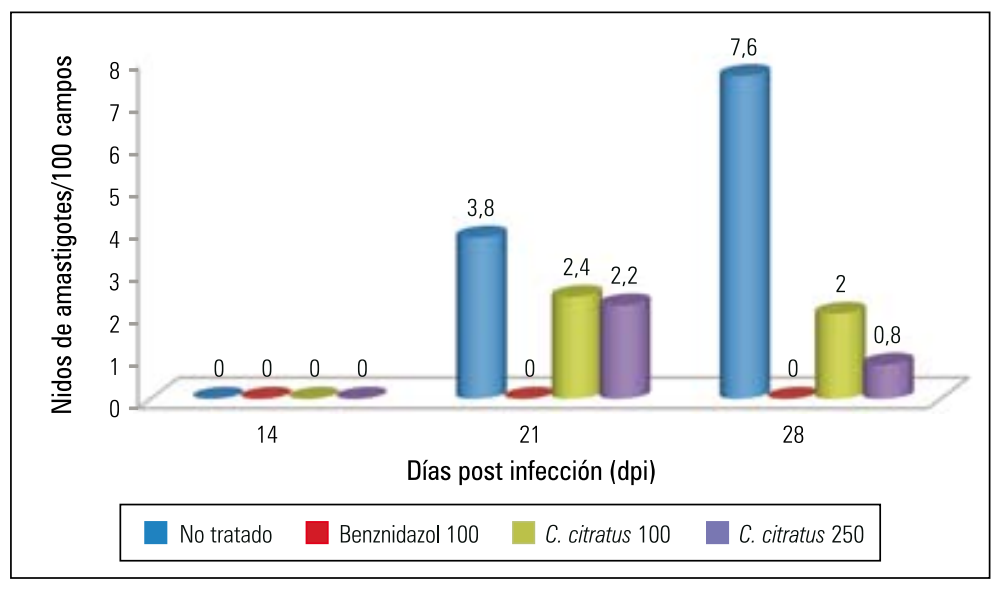

Figura 2. Nidos de amastigotes/100 campos en corazón de ratones infectados con $10^{4}$ tripomastigotes sanguíneos de T. cruzi y tratados con benznidazol y Cymbopogon citratus. En el 28 dpi se produjo una reducción significativa en el grupo tratado con 250 $\mathrm{mg} / \mathrm{kg}$ de $C$. citratus, desde 7,60 $\pm 5,55$ a 0,80 $\pm 1,19$ amastigotes $/ 100$ campos $(p<0,05)$.

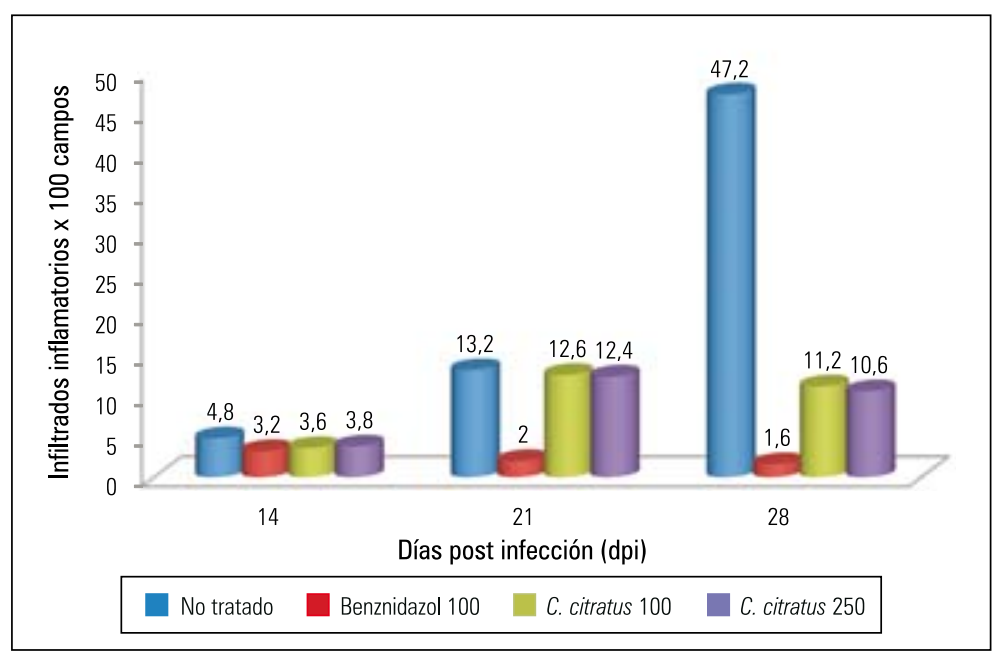

Figura 3. Infiltrados inflamatorios/100 campos en corazón de ratones infectados con $10^{4}$ tripomastigotes sanguíneos de T. cruzi y tratados con benznidazol y C. citratus. En el 28 dpi se produjo una reducción significativa en los grupos tratados con $100 \mathrm{y}$ $250 \mathrm{mg} / \mathrm{kg}$ de $C$. citratus $(p<0,001)$.
El número de infiltrados inflamatorios/100 campos se incrementó progresivamente en el grupo infectado y no tratado, siendo mayor en el $28^{\circ}$ dpi. El tratamiento con el aceite esencial de Cymbopogon citratus en dosis de $250 \mathrm{mg} / \mathrm{kg}$ produjo una reducción significativa de infiltrados inflamatorios en el $28^{\circ} \mathrm{dpi}$, de $47,20 \%+12,62$ a 10,60 + 1,67 ( p < 0,001) infiltrados inflamatorios/100 campos, mientras que con $100 \mathrm{mg} / \mathrm{kg}$ de C. citratus se redujo hasta $11,20 \pm 4,82(\mathrm{p}<0,001)$ (figura 3).

\section{DISCUSIÓN}

En la búsqueda de nuevas drogas alternativas para el tratamiento de protozoarios hemoflagelados, el aceite esencial de Cymbopogon citratus (DC) Stapf aparece como agente prometedor con actividad contra ciertos parásitos. Tanto este aceite esencial como su principal componente, el citral, mostraron actividad antiproliferativa sobre promastigotes y amastigotes axénicos y amastigotes intracelulares de Leishmania amazonensis, en donde los promastigotes sufrieron notables alteraciones morfológicas y ultraestructurales (36). Asimismo, se ha demostrado que este aceite esencial presentó acción inhibitoria in vitro sobre promastigotes de Leishmania chagasi, observándose alteraciones morfológicas drásticas del parásito, incluyendo hinchazón celular, acumulación de gotas de lípidos en el 
citoplasma e incremento del volumen acidocalcisoma ${ }^{(37)}$. Por otra parte, el aceite esencial de Cymbopogon citratus también mostró significativa actividad contra Plasmodium berghei en ratones, produciendo una supresión de la parasitemia de hasta $86,6 \%{ }^{(38)}$.

En nuestra investigación, el aceite esencial de Cymbopogon citratus (hierba luisa) produjo reducción del pico de parasitemia (figura 1) y reducción significativa del número de nidos de amastigotes e infiltrados inflamatorios en el tejido cardiaco (figuras 2 y 3), aunque el efecto no fue comparable al de la droga de referencia, el benznidazol, que en la dosis de $100 \mathrm{mg} / \mathrm{kg} /$ día produjo la reducción del 100\% tanto de tripomastigotes sanguíneos como de amastigotes tisulares. Estos resultados podrían guardar relación con el estudio in vitro de Santoro y col., el año $2007{ }^{\text {(39) }}$, quienes demostraron en Brasil que el aceite esencial de Cymbopogon citratus inhibió el crecimiento de epimastigotes y tripomastigotes de Trypanosoma cruzi e inhibió fuertemente la proliferación de amastigotes intracelulares. También, se ha demostrado actividad in vitro contra Trypanosoma brucei brucei ${ }^{(40)}$.

En la cuarta semana, se pudo apreciar una disminución de la parasitemia y de los nidos de amastigotes en todos los grupos (figuras 1 y 2), relacionado probablemente al control del crecimiento del parásito en el tejido que ejerce el sistema inmune por reclutamiento principalmente de células $\mathrm{CD}^{+}$, que destruyen los reservorios intracelulares y por macrófagos activados por interferon- $\gamma^{(41)}$. Asimismo, la quimioquina CCL2 participa en la reducción de la carga parasitaria, controlando la infiltración celular y la activación mononuclear durante la fase aguda de la infección por T. cruzi ${ }^{(42)}$. Por otra parte, los propios cardiomiocitos podrían contribuir a controlar el crecimiento del parásito y el influjo celular mediante la activación de la enzima óxido nítrico sintasa inducible (iNOS), citoquinas proinflamatorias y quimioquinas ${ }^{(43)}$.
La variación en la ganancia de peso corporal en el curso de los 28 dpi demostró que, a pesar que hubo una reducción del peso en los grupos tratados con el aceite esencial de Cymbopogon citratus, no hubo diferencias significativas al final del tratamiento (tabla 1), lo cual nos podría indicar una aparente falta de toxicidad del aceite esencial en las dosis utilizadas. Bañuelos y col., en $2006^{(44)}$, demostraron una menor ganancia de peso en ratones al administrar durante 30 días el aceite esencial de esta planta con el agua de bebida; en general, no observaron lesiones histopatológicas significativas y solo efectos irritantes leves en piel o mucosa esofágica, por el contacto prolongado. Se ha demostrado que este aceite esencial, en concentraciones menores a $100 \mu \mathrm{L} /$ $\mathrm{mL}$, no muestra citotoxicidad sobre células epidérmicas humanas de la línea HaCat ${ }^{(45)}$. También se ha demostrado la falta de citotoxicidad sobre macrófagos ${ }^{(36)}$. En otro estudio, la administración de este aceite con alimentos a ratas durante 60 días no mostró toxi$\operatorname{cidad}^{(46)}$.

El análisis químico del aceite esencial de Cymbopogon citratus (DC) Stapf realizado en Brasil, revela que el principal componente es el citral ${ }^{(47)}$, el cual es una mezcla de dos aldehídos monoterpénicos isoméricos, geranial y neral; al isómero trans- se le conoce como geranial o citral $\mathrm{A}$, y el isómero cis- es conocido como neral o citral B. Esto podría sugerir la posibilidad que el citral esté involucrado en la actividad anti-Trypanosoma cruzi, dado que se ha demostrado que diversas estructuras terpénicas de otras especies vegetales exhiben actividad contra uno o todos los estadíos de Trypanosoma cruzi ${ }^{(48-52)}$.

En conclusión, en las condiciones del experimento, el aceite esencial de Cymbopogon citratus (DC) Stapf tuvo efecto anti-Trypanosoma cruzi en ratones Balb/c, en lo referente a la disminución de la parasitemia, el número de nidos de amastigotes y los infiltrados inflamatorios.

\section{REFERENCIAS BIBLIOGRÁFICAS}

1. Ministerio de Salud. Dirección General de epidemiología. Boletín epidemiológico (Lima), 2006;15(33):5.

2. Brunton L, Lazo J, Parker K. En: Goodman \& Gilman (edit). Las Bases Farmacológicas de la Terapéutica. $11^{\text {a }}$ ed. Colombia: McGraw-Hill Interamericana. 2007:1049-62.

3. Negrelle R, Gomes E. Cymbopogon citratus (DC.) Stapf: chemical composition and biological activities Rev Bras PI Med. 2007;9(1):80-92.

4. Beyra A, León M, Iglesias E, Ferrándiz D, Herrera $\mathrm{R}$, Volpato $\mathrm{G}$ y col. Estudios etnobotánicos sobre plantas medicinales en la provincia de Camagüey (Cuba). Anales Jardin Botánico de Madrid. 2004;61(2):185-204.

5. Macia J, Garcia M, Vidaurre J. An ethnobotanical survey of medicinal plants commercialized in the markets of La Paz and El Alto, Bolivia. J Ethnopharmacol. 2005;97:337-50.

6. Mongkolsilp S, Pongbupakit I, Sae-Lee N, Sitthithaworn W. Radical scavenging activity and total phenolic content of medicinal plants used in primary health care. SWU J Pharm Sci. 2004;9(1):32-5.

7. Cápiro N, Sánchez A, Fonseca G, Baluja L, Borges E. Capacidad protectotora de Cymbopogon citratus DC Stapf ante el daño genético inducido por estrés oxidativo. Rev Cubana Invest Biomed. 2001;20(1):33-8.

8. Rao B, Shanbhoge R, Rao BN, Adiga S, Upadhya D, Aithal B, Kumar M. Preventive efficacy of hydroalcoholic extract of Cymbopogon citratus against radiation-induced DNA damage on V79 cells and free radical scavenging ability against radicals generated in vitro. Hum Exp Toxicol. 2009;28(4):195-202

9. Cheel J, Theoduloz C, Rodríguez J, SchmedaHirschmann G. Free radical scavengers and antioxidants from Lemongrass (Cymbopogon citratus (DC.) Stapf.). J Agric Food Chem. 2005;53(7):2511-7.

10. Tiwari M, Dwivedi U, Kakkar P. Suppression of oxidative stress and pro-inflammatory mediators by Cymbopogon citratus D Stapf extract in lipopolysaccharide stimulated murine alveolar macrophages. Food Chem Toxicol. 2010;48(10):2913-9.

11. Agbafor K, Akubugwo E. Hypocholesterolaemic effect of ethanolic extract of fresh leaves of Cymbopogon citratus (lemongrass). Afr J Biotechnol. 2007;6(5):596-8.

12. Adeneye A, Agbaje E. Hypoglycemic and hypolipidemic effects of fresh leaf aqueous extract of Cymbopogon citratus Stapf. in rats. J Ethnopharmacol. 2007;112(3):440-4.

13. Guzmán-Gutiérreza A, Balderasa J, Aguilarb A, Navarrete A. Sedative activity of some plants used in Mexico to treat insomnia. Rev Latinoamer Quim. 2009;37(3):243-51.

14. Sforcin JM, Amaral JT, Fernandes A Jr, Sousa JP, Bastos JK. Lemongrass effects on IL-1 beta and IL-6 production by macrophages. Nat Prod Res. 2009;23(12):1151-9.

15. Figueirinha A, Cruz MT, Francisco V, Lopes MC, Batista MT. Anti-inflammatory activity of Cymbopogon citratus leaf infusion in lipopolysaccharide-stimulated dendritic cells: contribution of the polyphenols. J Med Food. 2010;13(3):681-90.

16. Nwachukwu I, Allison L, Chinakwe E, Nwadiaro P. Studies on the effects Cymbopogon citratus, Ceiba pentandra and Loranthus bengwelensis extracts on species of dermatophytes. J Amer Sci. 2008;4(4):58-67. 
17. Wright SC, Maree JE, Sibanyoni M. Treatment of oral thrush in HIV/AIDS patients with lemon juice and lemon grass (Cymbopogon citratus) and gentian violet. Phytomed. 2009;16(2-3):118-24.

18. Okigbo R, Mmeka C. Antimicrobial effects of three tropical plant extracts on Staphyolococcus aureus, Escherichia coli and Candida albicans. Afr J Trad CAM. 2008;5(3):226-9.

19. Asaolu M, Oyeyemi O, Olanlokun J. Chemical compositions, phytochemical constituents and in vitro biological activity of various extracts of Cymbopogon citratus. Pakistan J Nutr. 2009;8(12):1920-2.

20. Suaeyun R, Kinouchi T, Arimochi H, Vinitketkumnuen $U$, Ohnishi $Y$. Inhibitory effects of lemon grass (Cymbopogon citratus Stapf) on formation of azoxymethane-induced DNA adducts and aberrant crypt foci in the rat colon. Carcinogenesis. 1997;18(5):949-55.

21. Puatanachokchai R, Kishida H, Denda A, Murata N, Konishi Y, Vinitketkumnuen U, Nakae D. Inhibitory effects of lemon grass (Cymbopogon citratus, Stapf) extract on the early phase of hepatocarcinogenesis after initiation with diethylnitrosamine in male Fischer 344 rats. Cancer Lett. 2002;183(1):9-15.

22. Orrego R, Leiva E, Cheel J. Inhibitory effect of three C-glycosylflavonoids from Cymbopogon citratus (Lemongrass) on human low density lipoprotein oxidation. Molecules. 2009;14:3906-13.

23. Abe S, Sato $Y$, Inoue S, Ishibashi H, Maruyama $\mathrm{N}$, Takizawa T, Oshima H, Yamaguchi H. [AntiCandida albicans activity of essential oils including Lemongrass (Cymbopogon citratus) oil and its component, citral]. Nippon Ishinkin Gakkai Zasshi. 2003;44(4):285-91.

24. Guerra M, Rodríguez M, Garcia G, Llerena C. Actividad antimicrobiana del aceite esencial y crema de Cymbopogon citratus (DC). Stapf. Rev Cubana Plant Med. 2004;9(2).

25. Irkin R, Korukluoglu M. Effectiveness of Cymbopogon citratus L. essential oil to inhibit the growth of some filamentous fungi and yeasts. Med Food. 2009;12(1):193-7.

26. Ohno T, Kita M, Yamaoka Y, Imamura S, Yamamoto T, Mitsufuji S, et al. Antimicrobial activity of essential oils against Helicobacter pylori. Helicobacter. 2003;8(3):207-15.

27. Viuda-Martos M, El-Nasser G, El Gendy S, Sendra E, Fernández-López J, El Razik K, et al. Chemical composition and antioxidant and anti-Listeria activities of essential oils obtained from some egyptian plants. J Agric Food Chem. 2010;58(16):9063-70.

28. Blanco M, Costa C, Freire A, Santos J, Costa M. Neurobehavioral effect of essential oil of Cymbopogon citratus in mice. Phytomed. 2009;16:265-70.

29. Silva MR, Ximenes RM, da Costa JG, Leal LK, de Lopes AA, Viana GS. Comparative anticonvulsant activities of the essential oils (EOs) from Cymbopogon winterianus Jowitt and Cymbopogon citratus (DC) Stapf. in mice. Naunyn Schmiedebergs Arch Pharmacol. 2010;381(5):415-26.

30. Viana GS, Vale TG, Pinho RS, Matos FJ. Antinociceptive effect of the essential oil from Cymbopogon citratus in mice. J Ethnopharmacol. 2000;70(3):323-7.
31. Pushpanathan T, Jebanesan, A, Govindarajan M. Larvicidal, ovicidal and repellent activities of Cymbopogon citratus Stapf (Graminae) essential oil against the filarial mosquito Culex quinquefasciatus (Say) (Diptera: Culicidae). Trop Biomed. 2006;23(2):208-12.

32. Jeong $H$, Sang $H$, Joong $D$, Hee $Y$, Yeon Yuk D, Tae J. Inhibitory effect of citral on NO production by suppression of iNOS expression and NF-KB activation in RAW264.7 cells. Arch Pharm Res. 2008;31(3):342-9.

33. Dudai N, Weinstein $Y$, Krup M, Rabinski T, Ofir R. Citral is a new inducer of caspase-3 in tumor cell lines. Planta Med. 2005;71(5):484-8.

34. Rojas J, Solís H, Palacios O. Actividad antiTrypanosoma cruzi in vitro de aceites esenciales de diez plantas medicinales. An Fac med. 2010;71(3):161-5.

35. Lahlou M. Methods to study the phytochemistry and bioactivity of essential oils. J Phytother Res. 2004;18:435-48.

36. Santin M, Oliveira A, Vataru C, Prado V, Piloto I, Ueda-Nakamura T. In vitro activity of the essential oil of Cymbopogon citratus and its major component (citral) on Leishmania amazonensis. Parasitol Res. 2009;105:1489-96.

37. Oliveira V, Moura D, Lopes J, de Andrade P, da Silva N, Figueiredo R. Effects of essential oils from Cymbopogon citratus (DC) Stapf., Lippia sidoides Cham., and Ocimum gratissimum L. on growth and ultrastructure of Leishmania chagasi promastigotes. Parasitol Res. 2009;104:1053-9.

38. Tchoumbougnang F, Zollo PH, Dagne E, Mekonnen Y. In vivo antimalarial activity of essential oils from Cymbopogon citratus and Ocimum gratissimum on mice infected with Plasmodium berghei. Planta Med. 2005;71(1):20-3.

39. Santoro G, Cardoso M, Guimaraez L, Freire J, Soares J. Anti-proliferative effect of the essential oil of Cymbopogon citratus (DC) Stapf (lemongrass) on intracellular amastigotes, bloodstream trypomastigotes and culture epimastigotes of Trypanosoma cruzi (Protozoa: Kinetoplastida). Parasitol. 2007;134:1649-56.

40. Habila N, Agbaji A, Ladan Z, Bello I, Haruna E, Dakare $\mathrm{M}$, et al. Evaluation of in vitro activity of essential oils against Trypanosoma brucei brucei and Trypanosoma evansi. J Parasitol Res. 2010:1-5.

41. Martin D, Tarleton R. Generation, specificity, and function of CD8p T cells in Trypanosoma cruzi infection. Immunol Rev. 2004:201:304-17.

42. Paiva CN, Figueiredo RT, Kroll-Palhares K. Silva AA, Silvério JC, Gibaldi D, et al. CCL2/MCP-1 controls parasite burden, cell infiltration, and mononuclear activation during acute Trypanosoma cruzi infection. J Leukoc Biol. 2009;86:1239-46.

43. Machado FS, Martins GA, Aliberti JC, Mestriner FL, Cunha FQ, Silva JS. Trypanosoma cruzi-infected cardiomyocytes produce chemokines and cytokines that trigger potent nitric oxide-dependent trypanocidal activity. Circulation. 2000;102(24);3003-8.

44. Bañuelos J, Alvarez J, Ruiz M, Barragán V, Rivas $E$, Meraz $C$, et al. Efectos tóxicos por la ingestión crónica de aceite esencial de Cymbopogon citratus
(DC) Stapf en ratones. XVII Semana de la Investigación Científica del CUCBA. 2006:722-5.

45. Koba K, Sanda K, Guyon C, Raynaud C, Chaumont J, Nicod L. In vitro cytotoxic activity of Cymbopogon citratus L. and Cymbopogon nardus L. essential oils from Togo. Bangladesh J Pharmacol. 2009;4:29-34.

46. Mishra A, Kishore N, Dubey N, Chansouria J. An evaluation of the toxicity of the oils of Cymbopogon citratus and Citrus medica in rats. Phytother Res. 1992;6:279-81.

47. Almeida L, Alves U, Martinazzo A, Álvares C Teixeira R, de Castro E. Evaluation of the chemical composition of Brazilian commercial Cymbopogon citratus (D.C.) Stapf samples. Molecules. 2008;13:1864-74.

48. Leite J, Oliveira A, Lombardi J, Filho J, Chiari E. Trypanocidal activity of triterpenes from Arrabidaea triplinervia and derivatives. Biol Pharm Bull. 2006;29(11):2307-9.

49. Araya J, Neira I, Da Silva S, Mortara R, Manque $\mathrm{P}$, Cordero E, et al. Diterpenoids from Azorella compacta (Umbelliferae) active on Trypanosoma cruzi. Mem Inst Oswaldo Cruz, Rio de Janeiro. 2003;98(3):413-8.

50. Sanchez A, Jimenez V, Sartor T, Tonn C, Garcia E, Nieto M. A novel icetexane diterpene, 5-epi-icetexone from Salvia gilliessi is active against trypanosoma cruzi. Acta Trop. 2006;98(2):118-24.

51. Cunha W, Crevelin E, Arantes G, Crotti A, Andrade M, Furtado M, et al. A study of the trypanocidal activity of triterpene acids isolated from Miconia species. Phytother Res. 2006;20(6):474-8.

52. Jimenez V, Brengio S, Giordano O, Tonn C, Sanchez M, Burgos M, et al. The trypanocidal effect of sesquiterpene lactones helenalin and mexicanin on cultured epimastigotes. J Parasitol. 2005;91(1):170-4.

Artículo recibido el 6 de diciembre de 2011 y aceptado para publicación el 10 de enero de 2012.

Trabajo financiado por el Vicerrectorado de Investigación de la Universidad Nacional Mayor de San Marcos.

Los autores declaran no tener ningún conflicto de interés para la publicación de este artículo.

Autor corresponsal:

Dr. Juan Rojas Armas

Instituto de Investigaciones Clínicas, Hospital Nacional Dos de Mayo

Av. Grau cuadra 13, Parque Historia de la Medicina, Lima 1, Perú

Correo electrónico: jprojasarmas@yahoo.com 